\title{
Critical indices of random planar electrical networks
}

\author{
J.S. Espinoza Ortiz \\ Departamento de Física,Universidade Federal de Goiás, Catalão, GO 75700-000, Brazil
}

\author{
Gemunu H. Gunaratne \\ Department of Physics, University of Houston, Houston, TX 77204, USA and \\ The Institute of Fundamental Studies, Kandy 20000, Sri Lanka
}

(Received on 6 February, 2009)

\begin{abstract}
We propose a new method to estimate the percolation threshold $p_{c}$ and the critical index $t$ associated with strength reduction in networks of random fused conductors. It relies on a recently proposed expression for the yield strength of a network as a function of the probability $p$ that each element is removed from it. The values of critical indices are confirmed using finite size scaling. Further, we systematically study effects of different damage modalities, which are chosen to reflect age-related changes in the porous inner segments of human bone. In particular, we find that $p_{c}$ and $t$ depend on damage modalities.
\end{abstract}

Keywords: Random networks; Fused conductors;Human bone; Strength reduction; Critical index

\section{INTRODUCTION}

Random resistor networks are used to model a variety of physical phenomena ranging from properties of inhomogeneous media [1-6], metal insulator transitions [7], dielectric breakdown [8-11], the role of percolation in weak and strong disorder [12-14], and the strength of trabecular bone [1517]. Studies discussed in this paper, although only for planar networks, are motivated by the last example.

Large bones of the human skeleton consist of an outer compact shaft (cortex) of thickness $2-4 \mathrm{~mm}$, and an inner porous region (trabecular architecture) whose structure is reminiscent of disordered cubic networks with elements of length $1 \mathrm{~mm}$ and thickness $0.1 \mathrm{~mm}$. With aging, the cortex weakens due to the accumulation of fractures, and the trabecular bone plays increasingly important role in load carrying [16]. Consequently, there is intense interest in discovering how trabecular bone weakens, and identifying non- invasive diagnostics of its strength. Two factors dominate its age-related metamorphoses. They are random damage which occurs during daily activity and the preferential regeneration of bone that is under high levels of stress/strain $[15,16]$. One of the primary mechanisms for bone loss is through the removal of individual trabecular elements [17]. It is initiated by fractures that sever a trabecular element and is compounded by the its decay because the element no longer carries a load. Second, the fact that axial loads on a bone are typically larger than off-axial loads means that the trabecular elements in the axial direction are preferentially strengthened through regeneration. Consequently, the level of anisotropy of trabecular bone networks increases with aging [18]. The third factor motivating our studies relies on the following observation; mechanical experiments on ex vivo bone samples have shown that trabecular networks from subjects fracture at a fixed level of strain [18], even though the corresponding fracture stresses vary significantly with age. This suggests the use of a strain-based fracture criterion for individual trabecular elements [17].

We consider here, the problem of failure in a disordered network of fused conducting elements under the influence of an electrical field. Although this scalar framework is simpler than the vector models of mechanical failure of elastic networks, both classes are expected to have similar scaling properties close to the percolation threshold [19-22]. The primary question we address is how the strength of a network is effected by different types of damage it incurs. Age-related loss of trabecular elements is modeled by a random removal of conductances from the network. The analog of the strainbased fracture criterion of bone is a voltage-based fusing of each electrical element [23]. Increasing levels of anisotropy of trabecular bone with aging is modeled by corresponding changes in the electrical network.

In Ref. [23], we studied the breakdown of fused electrical networks. Specifically, we determined how the yield strength, i.e., the largest potential that the network can be subjected to before the first electrical element fuses, decreases as elements are removed from the network. We first used Green's function techniques to obtain an expression for the reduction in yield strength of an infinite hypercubic network due to a single fracture of length $\kappa$. We next considered random removal of electrical elements, and proposed a generalization to this expression (Eqn. [1]), which provides the yield strength as a function of the probability $p$ that an element is removed from the network. Unlike previously proposed scaling expressions, which are valid only near the percolation threshold, this expression was seen to hold for all values of $p$. We thus inquire if it can be used to a obtain a good estimate for the critical index [2]. Since this technique has not been proven, we check our estimates of the critical index using the finite-size-scaling ansatz. We then test if the critical indices remain unchanged when other criteria are used to degrade the network. These criteria are chosen to model age-related damage to trabecular bone. For example, the fact that the degradation of trabecular bone is highly anisotropic [17], motivates the study of the critical index of electrical networks subjected to anisotropic removal of electrical elements. Further, trabecular elements are not only removed with aging, but those remaining ones degrade on average. The corresponding study of electrical networks would require reducing the strength of elements at a rate proportional to the fraction of elements that are removed. Finally, the observation that some trabecular elements remaining in a bone can strengthen due to extra loads created by thinning of the network [17] suggest an analysis of networks whose electrical elements are changed in a range $(1-\varepsilon, 1+\varepsilon)$. We 
study changes in the percolation threshold and the critical index under each of these scenarios. As discussed in Ref. [24], linear response functions can be used as a diagnostic of bone strength if the percolation threshold of the trabecular network is known.

The model and the expression which relate the reduction of yield strength to statistical properties of a network is set up in section II. In Section III, we study effects of several damage modalities of the network. Section IV provides conclusions.

\section{THE MODEL}

We consider square networks of fused conductors, each of which fail when the potential difference across them reaches a pre-set value; i.e., the breakdown current of an element is proportional to its conductance. Typically, failure of an element increases currents on neighboring conductors, enhancing the likelihood of their failure [25, 26]. We study the yield point at which the external current initiates the first failure. The peak currents on a network show similar scaling behavior [27].

Consider first, a complete square network of size $M \times M$, with the top and the bottom edges at potentials $V_{0}$ and 0 respectively. Assume that each electrical element in the network fails when the potential difference across it reaches a value $V_{b}$. We can calculate the current $I(0)$ flowing through the network using Kirchhoff's laws. As $V_{0}$ increases, currents through the conductors, as well as $I(0)$, will increase until the yield point $I(0)=I_{\text {yield }}(0)$, when the first failure of an electrical element is induced.

Next consider a (degraded) network obtained by removing electrical elements with a probability $p$. Denote the yield current of this network by $I_{y i e l d}(p)$. (Note that the definition of $I_{\text {yield }}(0)$ in the previous paragraph is consistent with this.) Typically, $I_{\text {yield }}(p)$ decreases with increasing $p$, and vanishes as $\left(p_{c}-p\right)^{t}$ when $p$ approaches the percolation threshold $p_{c}(=0.5$ for isotropic square networks). $t$ is the critical index, with reported values between 1.1 and 1.43 under different scenarios of damage and symmetries of the network [2836].

There have been several proposals for the reduction of strength of a network due to a random removal of element $[19,20]$. Here we test a recently proposed expression for $I_{\text {yield }}(p)$ [23]:

$$
\tau(p) \equiv \frac{I_{\text {yield }}(p)}{I_{\text {yield }}(0)}=\frac{1}{1+a_{1} z^{t / 2}+a_{2} z^{t}},
$$

where $z=\log (N) / \log \left(\frac{p_{c}}{p}\right)$. Here, $N\left(=M^{2}\right)$ is the number of nodes in the original network, $p_{c}$ is the bond percolation threshold for the class of network considered, and $a_{1}$ and $a_{2}$ are constants that depend on the model and damage modalities, see below. The form of this expression was deduced from the following observations [23]: (1) A calculation which shows that the reduction in yield strength of a network due to a single fracture of length $\kappa$ is

$$
\tau(\kappa) \approx \frac{1}{1+a_{1} \kappa^{1 / 4}+a_{2} \kappa^{1 / 2}} .
$$

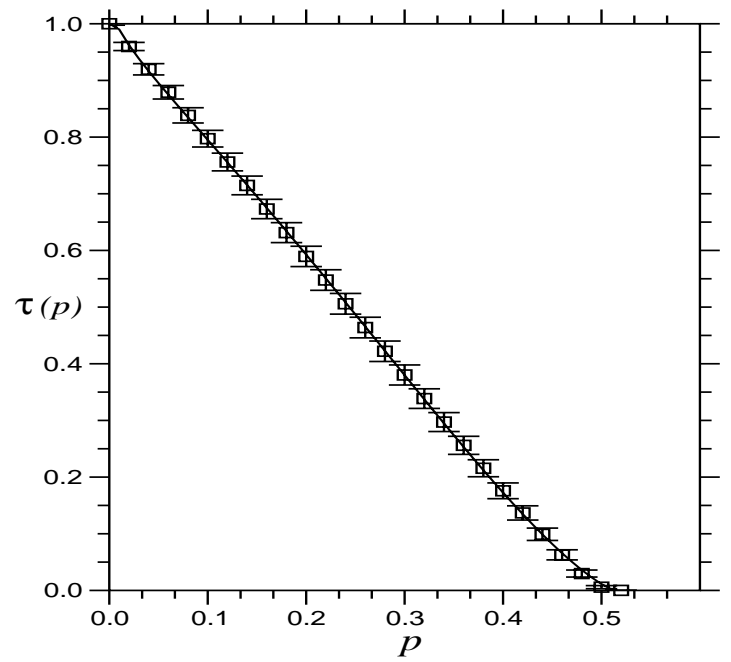

FIG. 1: Strength reduction in a $160^{2}$ network due to a random isotropic removal of a fraction $p$ of conductances, averaged over one thousand trials. Numerical results are shown by boxes along with the statistical error, while those obtained by fitting to expression (1) are shown as a continuous line.

(2) the expected size of the largest fracture when elements are removed with a probability $p$ is $\ln M / \ln (1 / p)$. (3) As $p \rightarrow$ $p_{c}$, the yield strength vanishes as $\tau(p) \rightarrow\left(p_{c}-p\right)^{t}$. Equation (1) is valid throughout the range $p \in\left[0, p_{c}\right)$ [23], and we use it to estimate $p_{c}$ and the critical index $t$. We then confirm the value of $t$ using finite size scaling.

The yield current for a given network is computed as follows: given the conductance $\sigma_{i}$ of all electrical elements in the network and the potential $V_{o}$ of the top layer of nodes, we use Kirchhoff's laws to determine the currents $i_{k}$ through each element and the potential differences $v_{k}$ across them. We denote the largest of the latter by $V_{\max }$. The network will "yield" when $V_{\max }$ reaches $V_{b}$. Since $V_{\max }$ and $I(p)$ grow linearly with $V_{0}$, the yield current is $I_{\text {yield }}(p)=I(p) \times V_{b} / V_{\text {max }}$.

\section{RESULTS AND DISCUSSIONS}

Our computations used networks with sizes $M=$ $20,30,40,50,70,80,90,120,140$ and 160 , and sample sizes of 10,000 for $M=20,30,40,50$, sample size of 2,500 for $M=70,80,90$ and finally sample size of 1,000 for $M=120,140,160$. Figure 1 shows the behavior of $\tau(p)$ for the $160 \times 160$ networks, where the error bars show standard errors. Although fluctuations $\delta \tau$ in $\tau(p)$ decrease as $p \rightarrow p_{c}$, the relative fluctuations $(\delta \tau / \tau)$ increase. The solid line shown in Figure 1 represents the best fit to Equation (1) with parameters $a_{1}, a_{2}, p_{c}$ and $t$ in a $160^{2}$ conducting network. To determine their values, $a_{1}=-0.1043 \pm 0.005, a_{2}=0.061 \pm$ $0.003, p_{c}=0.513 \pm 0.002$ and $t=1.228 \pm 0.015$, we used the Lavenberg-Marquadt method to implement the nonlinear fit [37]. We must fit $t$ and $p_{c}$ because they depend on the size of the network.

Next, we determine how $p_{c}$ and $t$ change with the network 


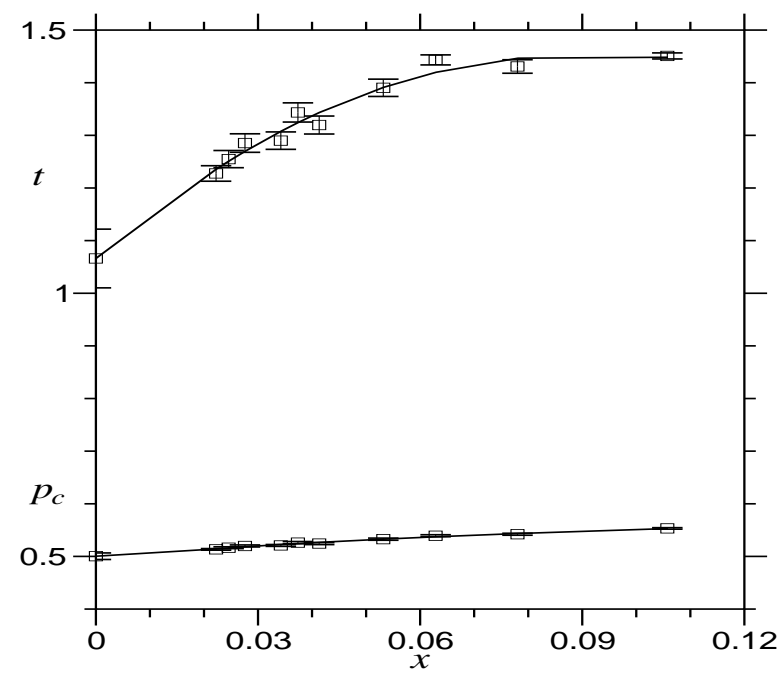

FIG. 2: Variation of parameters $t$ and $p_{c}$ versus the inverse correlation length $\left(x=M^{-0.75}\right)$, for networks of size $M=20$ to $M=160$. All elements in the initial networks have conductance of 1 unit. Note that, both critical values for $t(=1.066 \pm 0.056)$ and $p_{c}(=0.500 \pm 0.006)$ are getting in the limit when $x \rightarrow 0$.

size. We express the parameters as a function of $x=M^{-1 / v}$ where $v(=4 / 3$ for $2 \mathrm{D}$ square networks $)$ is the universal correlation length exponent $[38,39]$; i.e, $x$ is the inverse of the mean size of the largest domain in a network of size $M \times M$. Figure 2 shows the values of $t(x)$ and $p_{c}(x)$, along with the error estimates.

We estimate the value of $t(0)$ corresponding to an infinite network by first approximating $t(x)$ by a rational function $f(x) / g(x)$ (where $f(x)$ and $g(x)$ are polynomials of order 3 and 2 respectively) and extrapolating to $x=0$. The values of the extrapolation corresponding to Figure 2 are $p_{c}(0)=0.500 \pm 0.006, t(0)=1.066 \pm 0.056, a_{1}(0)=$ $-0.106 \pm 0.015$ and $a_{2}(0)=0.060 \pm 0.004$. The error estimate includes both errors at each $M$ and those due to the extrapolation [37].

We now validate our results using finite size scaling to independently estimate $t(0)$. According to the finite size scaling ansatz, for the correct $t(0)$, the relationship between the rescaled variables $\bar{\tau}=M^{t(0) / v} \times \tau$ and $\zeta=M^{-1 / v}\left(p_{c}-p\right)$ is independent of the system size $M$ [40]. Although the finite size scaling ansatz need hold only for $p \rightarrow p_{c}$ (and $0<M^{-1 / v}<1$ ), we find that the data collapses to a scaling function $\bar{\tau}(\zeta)$ over the entire range $p \in\left[0, p_{c}\right]$; see Figure 3 (left side). We then determine the best value for $t(0)$ : for any chosen value of $t(0)$, we approximate the scaling function $\bar{\tau}(\zeta)$ by a rational function $\bar{f}(x) / \bar{g}(x)$ (where $\bar{f}(x)$ and $\bar{g}(x)$ are polynomials of order 3 and 2 respectively) and estimate the deviation of the data $\left(\zeta_{k}, \bar{\tau}_{k}\right)$ from the scaling function by $\chi^{2}=\sum_{k}\left(\bar{\tau}_{k}-\bar{\tau}\left(\zeta_{k}\right)\right)^{2}$. Here, the sum is over all available networks. Figure 3 (right side) shows the $\chi^{2}$ as a function of $t(0)$; the best estimate, which we assume minimizes $\chi^{2}$, is $t_{F S S}=1.07 \pm 0.10$, where the error estimate corresponds to doubling the $\chi^{2}$ value. This estimate agrees with that value we obtained from Equation (1). These processes are followed in calculations for other classes of networks discussed below.

Next we consider networks from which elements are removed anisotropically. We begin with a square network of unit conductances and remove elements in the horizontal and vertical directions with probabilities $p_{h}=\beta p$ and $p_{v}=p$. Analysis of such networks for $\beta=2.0$ using Equation (1) gives $p_{c}=0.3383 \pm 0003, t=1.33 \pm 0.02$. Similarly, for $\beta=1 / 2, p_{c}=0.68 \pm 0.01, t=1.04 \pm 0.06$ (See Figure 4). Finite size scaling for the two cases estimates give $t_{F S S}=1.30 \pm 0.10$ and $t_{F S S}=1.05 \pm 0.10$ respectively, in good agreement with results obtained from the use of Equation (1). The estimates for $p_{c}$ for multiple $\beta$ 's, shown in Figure 4, agree with theoretical results for anisotropic networks [1].

Next, we consider the following process with a two-step degradation. Random removal of conductors is followed by multiplying the conductivity of the remaining conductors by a random number in the range $(1-\varepsilon, 1+\varepsilon)$; where $\varepsilon \in(0,1)$. Note that, the isotropic case is retrieved for $\varepsilon=0$. This process is meant to model age-related changes in trabecular bone. Specifically, while elements of the trabecular network are removed with aging, those remaining can become weaker or stronger - through bone regeneration- depending on the mean loads they carry [15]. We find that the critical index depends on the value of $\varepsilon$. We conduct our analysis for $\varepsilon=0.00,0.25,0.50,0.75,0.85,0.95$ and 1.0 , and at each $\varepsilon$, for network sizes considered earlier. Although the value of the critical point $p_{c}$ is independent of $\varepsilon$ (Figure 5), and the critical exponent increases with $\varepsilon$ (for $\varepsilon>0.5$ ) (Figure 5), consistent with results for finite size scaling.

Next, we consider networks where electrical elements that remain in the network are randomly degraded as $p$ increases. Once again, this problem is related to degradation of porous bone with aging [41], namely the simultaneous loss of trabecular elements and reductions of the average thickness of the remaining elements. In modeling this dual process, we assume a proportionality between the two types of degradation. Specifically, we consider networks whose conductance and breakdown decrease by a factor $(1-\alpha p)$, where as be-

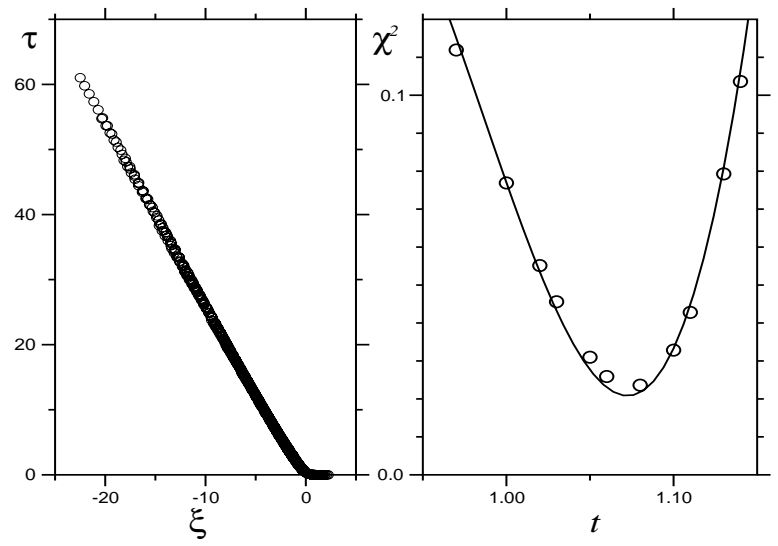

FIG. 3: The left side of the figure shows the scaling function $(\tau)$ behavior as a function of the network correlation length $(\xi)$, and its $\chi^{2}$ error as a function of $t$ for fixed unit conductances in the network (right side). The minimum of $\chi^{2}$ is $t$, gives the critical exponent. 


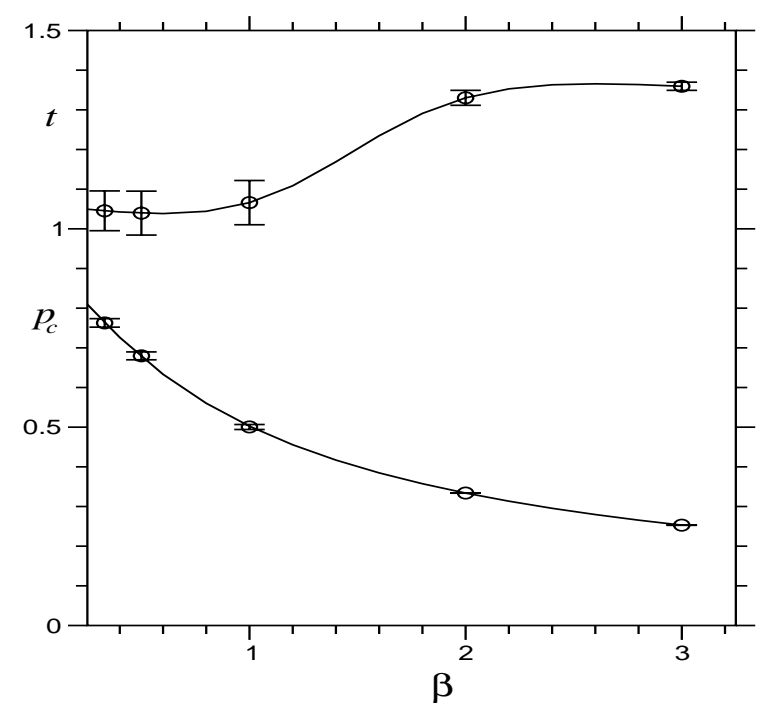

FIG. 4: Values of $t(\beta)$ and $p_{c}(\beta)$ with $\beta$, for anisotropic removal of conductance. Elements in the vertical and horizontal directions are removed with probabilities $p$ and $\beta p$.

fore, $p$ is the probability for an element to be removed from the network. Once again, as earlier, we analyzed network sizes ranging from $M=20$ to $M=160$ (Figure 6). For $\alpha=$ 1.0 we find that $p_{c}=0.509 \pm 0.005, t=1.29 \pm 0.03, a_{1}=$ $-0.123 \pm 0.006$ and $a_{2}=0.067 \pm 0.003$. The estimated critical exponent using finite size scaling is $t_{F S S}=1.29 \pm 0.10$.

We end this Section by briefly discussing a clinically relevant issue. The primary non-invasive diagnostic used to estimate bone strength and the need for therapy is bone density $[18,41]$. In using it, there is an implicit assumption that the relationship between bone strength and density is independent of the damage modality of bone. The fact that the per-

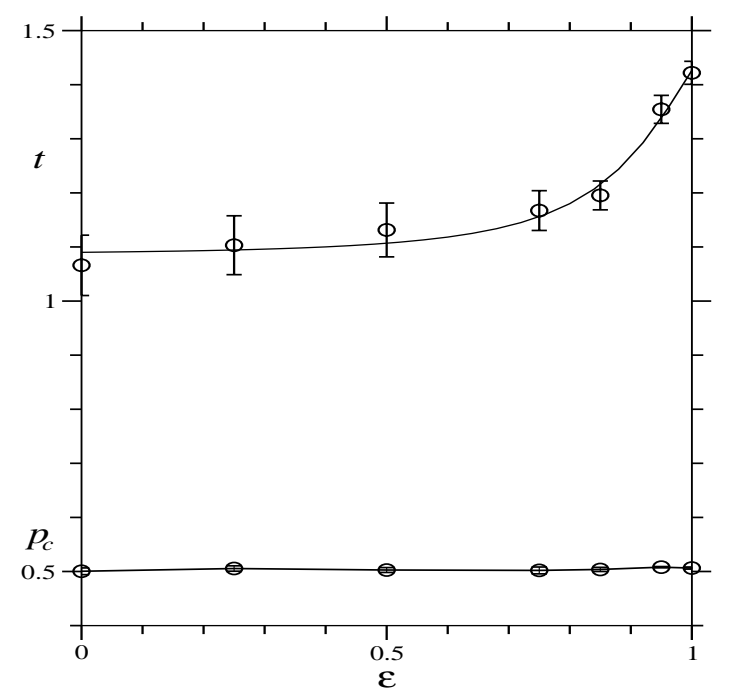

FIG. 5: Behavior of $t(\varepsilon)$ and $p_{c}(\varepsilon)$ with $\varepsilon$, when conductances of the initial network are chosen randomly within $(1-\varepsilon, 1+\varepsilon)$. Bonds removal is isotropic ( $p_{c}$ remains at 0.5 ).

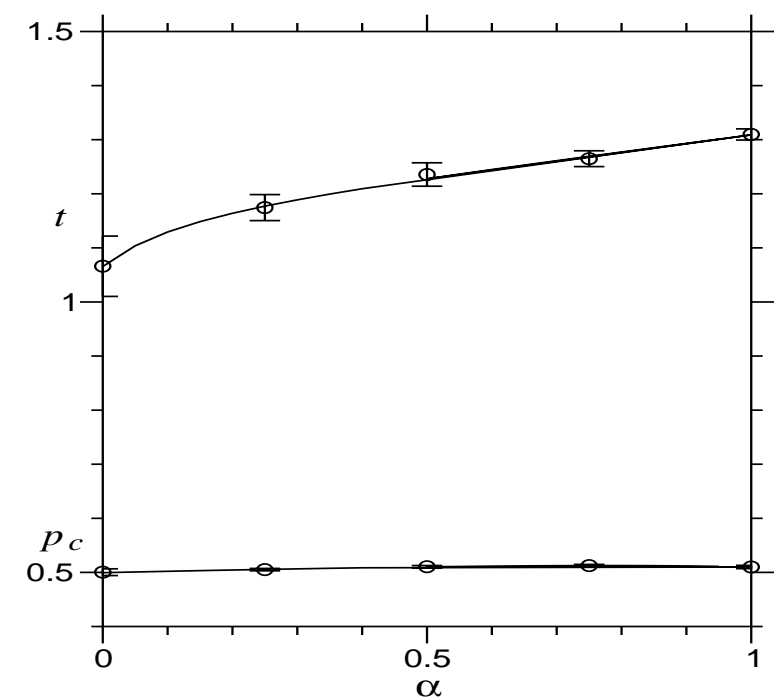

FIG. 6: Variations in parameters $t(\alpha)$ and $p_{c}(\alpha)$. When elements are removed from the network with probability $p$, the conductance of those remaining are reduced simultaneously by a factor $(1-\alpha p)$. The critical fraction removed elements remain 0.5.

colation threshold and the critical index for $\tau(p)$ depend on the modality of damage brings the validity of this assumption into question. To test it, we study two distinct degradations of networks, see Figure 7. The panel on the right shows the $\tau(p)$ for the anisotropic decay with $\beta=3.0$, while that on the left shows the relationship when conductors are randomly removed from the network while the remaining elements are reduced in strength by a factor $(1-0.75 p)$. Clearly, the relationship between $\tau(p)$ and $p$ (and hence very likely the relationship between $\tau(p)$ and bone density) depends on the damage modality. This observation suggests bone density scans, although widely used, may not be a reliable surrogate for bone strength.

\section{CONCLUSIONS}

We have computed percolation threshold $p_{c}$ and critical index $t$ for square networks of conductances under different damage modalities. These studies are motivated by types of age-related damage in human trabecular bone, and the need to assess their effects on bone strength. The computations are based on the expression (1) which was shown to hold for values of $p \in\left[0, p_{c}\right)$ [23]. We validate the values of $t$ using finite size scaling. For isotropic removal of conductances, we found $t=1.07 \pm 0.06$ in agreement with the value $t=1.1$ reported by Kirkpatrick [30], Straley [42, 43], and Stinchcombe and Watson [44]. However, it is different from the values obtained using real space renormalization group methods [34, 44-49] and low density series expansion [5052]. Series expansion has also been used to compute numerically the critical exponent [53], where for $d=2$ the best estimate is $t=1.299 \pm 0.002$. Many of these discrepancies have already been discussed by Straley [42].

For anisotropic networks, we compare our results to those 

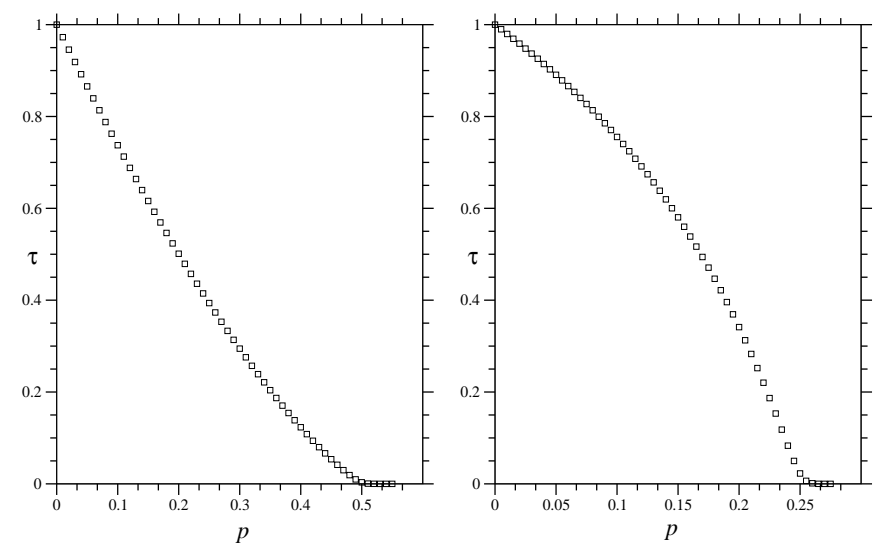

FIG. 7: The deviation from linearity of the strength reduction due to the removal of a fraction of conductance in a $160^{2}$ network. The right side shows the anisotropic removal of conductance with probabilities $p$ and $3.0 p$ in the vertical and horizontal direction. The left side corresponds the case where the elements are randomly removed with probability $p$ meanwhile the ones remaining are simultaneously reduced by a factor $(1-0.75 p)$. of experimental and computational results by Han, Lee and Lee [54] and Smith and Lobb [55]. Both groups found that $t=1.3$ when $p=0.33$. Figure 4 shows that for $p=0.33$, the parameter $\beta$ is 1.94 and $t=1.31$, in agreement with these previous analyses. Further, the values of $p_{c}(\beta)$ for anisotropic removal of conductances (Figure 4 ) is consistent with theoretical results of Redner and Stanley [1].

Perhaps our most interesting observation is the dependence of the critical index of the conductance network on the damage modality. It would be of interest to develop a renormalization group analysis to understand this variation. We have, thus far, not been successful in identifying such a theory.

\section{Acknowledgments}

The authors would like to thank Dr. K.E. Bassler for useful discussions. This work was partially funded by the National Science Foundation through grants DMS-0607345 and CMMI-0709293, the Institute of Space Science Operations and the ICSC-World Laboratory.
[1] S. Redner and H. E. Stanley, J. Phys. A: Math. Gen. 12, 1267 (1979).

[2] S. Redner, Review article for the Encyclopedia of Complexity and System Science (Springer Science), in Press, in arXiv:0710.1105, 27 pages (2007).

[3] M. Sahimi and J. D. Goddard, Phys. Rev. B 32, 1869 (1985).

[4] J. W. Chung, A. Ross, J. Th. De Hosson and E. van der Giessen, Phys. Rev. B 54, 15094 (1996).

[5] P. Ray and B. K. Chakrabarti, Phys. Rev. B 38, 715 (1988).

[6] Y. Kantor and I. Webman, Phys. Rev. Lett. 52, 1891 (1984).

[7] V. K. S. Shante and S. Kirkpatric, Adv. Phys. 20, 325 (1971).

[8] H. Takayasu, Phys. Rev. Lett. 54, 1099 (1985).

[9] J. C. Dyre and Th. B. Schoder, Rev. of Modern Phys. 72, 873 (2000).

[10] A. Hansen, E. L. Hinrichsen and S. Roux, Phys. Rev. B 43, 665 (1991).

[11] A. Hansen, E. L. Hinrichsen and S. Roux, Phys. Rev. Lett. 66, 2476 (1991).

[12] C. Tsallis and S. Redner, Phys. Rev. B 28, 6603(R) (1983).

[13] Zhenuhua Wu, Eduardo López, Sergey V. Buldyrev, Lídia A. Braunstein, Shlomo Havlin, H. Eugene Stanley, Phys. Rev. E 71, 045103(R) (2005).

[14] Guanlian Li, Lídia A. Braunstein, Sergey V. Buldyrev, Shlomo Havlin, H. Eugene Stanley, Phys. Rev. E 75, 04501(R) (2007).

[15] Y. C. Fung, Biomechanics: Mechanical Properties of Living Tissue (Springer - Verlag, N. Y., 1993).

[16] K. G. Faulkner, J. Bone Miner. Res. 15, 183 (2000).

[17] G. H. Gunaratne, C. S. Rajapakse, K. E. Bassler, K. K. Mohanty and S. J. Wimalawansa, Phys. Rev. Lett. 88, 68101 (2002).

[18] E. F. Morgan and T. M. Keaveny, J. Biomech. 34, 569 (2001).

[19] B. K. Chakrabarti and L. G. Benguigui, Statistical Physics of Fracture and Breakdown in Disordered Systems (Oxford University Press, Inc. N. Y., 1997).

[20] P. M. Duxbury, P. D. Beale and P. L. Leath, Phys. Rev. Lett. 57, 1052 (1986).
[21] P. M. Duxbury, P. D. Beale and P. L. Leath, Phys. Rev. B 36, 367 (1986).

[22] H. J. Herrmann, A. Hansen and S. Roux, Phys. Rev. B 39, 637 (1989).

[23] J. S. Espinoza Ortiz, Chamith S. Rajapakse and Gemunu H. Gunaratne, Phys. Rev. B 66, 144203 (2002).

[24] G. H. Gunaratne, Phys. Rev. E 66, 061904 (2002).

[25] J. W. Esam, Rep. Prog. Phys. 43, 53 (1980).

[26] D. Stauffer and A. Aharony, Introduction to Percolation Theory (Taylor and Francis, London, 1992).

[27] P. M. Duxbury, R. A. Guyer and J. Machta, Phys. Rev. B 51, 6711 (1995).

[28] C. J. Lobb and D. J. Frank, Phys. C 12, L827 (1979).

[29] S. Kirkpatrick, Rev. Mod. Phys 45, 574 (1973).

[30] S. Kirkpatrick, Proceeding on the Summer School on III Condensed Matter (North Holland, Amsterdam, 1978).

[31] Y. Gefen, A. Aharony, B. B. Mandelbrot and S. Kirkpatrick, Phys. Rev. Lett. 47, 1771 (1981).

[32] L. Benguigui, Phys. Rev. B 34, 8176 (1986).

[33] B. I. Halperin, S. Feng and P. N. Sen, Phys. Rev. Lett. 54, 2391 (1985).

[34] D. J. Frank and C. J. Lobb, Phys. Rev. B 37, 302 (1988).

[35] B. I. Halperin, Physica D 38, 179 (1989).

[36] M. Sahimi, Application of Percolation Theory (Taylor and Francis, London, 1994).

[37] W. H. Press, S. A. Teukolsky, W. T. Vetterling and B. P. Flannery, Numerical Recipes - The Art of Scientific Computing (Cambridge University Press, 1988).

[38] M. P. M. den Nijs, J. Phys. A 12, 1857 (1979).

[39] Yu A. Dzenis and S. P. Joshi, Phys. Rev. B 49, 3566 (1994).

[40] M. E. J. Newman and G. T. Barkema, Monte Carlo Methods in Statistical Physics (Oxford University Press, 2001).

[41] L. Mosekilde, Bone 9, 247 (1988).

[42] J. P. Straley, J. Phys. C 10, 1903 (1977).

[43] J. P. Straley, Phys. Rev. B 15, 5733 (1977).

[44] R. B. Stinchcombe and B. P. Watson, J. Phys. C 9, 3221 
(1976).

[45] M. Stephen, Phys. Rev. B 17, 4444 (1978).

[46] A. B. Harris, S. Kim and T. C. Lubensky, Phys. Rev. Lett. 53, 743 (1984).

[47] O. Stenull, H. K. Janssen and K. Oerding, Phys. Rev. E 59, 4919 (1999).

[48] J. Bernasconi, Phys. Rev. B 18, 2185 (1976).

[49] P. J. Reynolds, W. Klein and H. E. Stanley, J. Phys. C 10, L167 (1977).

[50] R. Fisch and A. B. Harris, Phys. Rev. B 18, 416 (1978).
[51] J. Adler, J. Phys. A: Math. Gen. 18, 307 (1985).

[52] J. Adler, Y. Meir, A. Aharony, A. B. Harris and L. Klein, J. Stat. Phys. 58, 511 (1990)

[53] J. M. Normand, H. J. Herrmann and M. Hajjar, J. Stat. Phys. 52, 441 (1988).

[54] K. H. Han, J. O. Lee and Sung-I K Lee, Phys. Rev. B 44, 6791 (1991).

[55] L. N. Smith and C. J. Lobb, Phys. Rev. B 20, 3653 (1979). 